\title{
A targeted needs assessment to improve referral patterns for palliative radiation therapy
}

\author{
Sean M. Parker ${ }^{1}$, Randy L. Wei ${ }^{2}$, Joshua A. Jones ${ }^{3}$, Malcolm D. Mattes ${ }^{4}$ \\ ${ }^{1}$ School of Medicine, West Virginia University, Morgantown, WV, USA; ${ }^{2}$ Memorial Radiation Oncology Medical Group, Fountain Valley, CA, USA; \\ ${ }^{3}$ Department of Radiation Oncology, Perelman School of Medicine, Philadelphia, PA, USA; ${ }^{4}$ Department of Radiation Oncology, West Virginia \\ University School of Medicine, Morgantown, WV, USA \\ Correspondence to: Malcolm D. Mattes, MD. Department of Radiation Oncology, West Virginia University, PO Box 9234, One Medical Center Drive, \\ Morgantown, WV, USA. Email: malcolm.mattes@gmail.com.
}

\begin{abstract}
Radiation therapy (RT) can effectively palliate a variety of symptoms in patients with metastatic cancer, using relatively low doses that infrequently cause major side effects. However, palliative radiation is often underutilized and sub-optimally implemented. In this study, we surveyed the Society of Palliative Radiation Oncology (SPRO) membership to identify barriers to appropriate referral for palliative RT that they encounter in their practice, and identify specific groups of physicians who radiation oncologists believed would benefit most from further education on when to refer patients. A total of 28 radiation oncologists responded to the survey with a response rate of $20.5 \%$. On average, participants felt that referrals for palliative RT were inappropriately delayed 46.5\% [standard deviation (STD) 20.2\%] of the time. The most common barrier to referral for medical oncologists was thought to be potential interference with systemic therapy (33\%); for primary care physicians and surgeons it was a lack of knowledge about the benefit (42\%), and for palliative care physicians it was concern for patient convenience (25\%). For brain metastases and spinal cord compression radiation oncology was felt to be part of the initial referral sequence more than $50 \%$ of the time, but less so for thoracic airway obstruction/bleeding (38\%), esophageal obstruction (16\%), or urinary obstruction/bleeding $(8 \%)$, where another subspecialist was more often consulted first. Primary care, geriatric medicine, and emergency medicine were considered among the least knowledgeable specialties about palliative radiation. These hypothesis-generating findings can guide approaches to improve referral patterns for this important aspect of supportive care.
\end{abstract}

Keywords: Cancer; palliative; radiation; referral

Submitted Mar 11, 2019. Accepted for publication Jul 31, 2019.

doi: 10.21037/apm.2019.08.02

View this article at: http://dx.doi.org/10.21037/apm.2019.08.02

\section{Introduction}

Radiation therapy (RT) can effectively palliate a variety of symptoms in patients with cancer, using relatively low doses of radiation that infrequently cause major side effects $(1,2)$. Common indications may include tumor-induced pain, bleeding, obstruction, or neurologic symptoms (3). Unfortunately, palliative RT is either not utilized at all or administered too late for many patients, in large part because delivering treatment depends upon appropriate referrals to radiation oncologists when a patient is symptomatic but still has sufficient life expectancy to benefit from treatment (4-7). Medical oncologists are the most common source of these referrals, since they are frequently following these patients closely as they give systemic therapy. However, physicians from many other specialties also encounter symptomatic patients with cancer in both the outpatient and inpatient setting, and can make direct referrals to radiation oncology. If these physicians also have a baseline understanding of indications for palliative radiation and feel empowered to refer patients, it would help expedite alleviation of their patients' symptoms and maximize quality of life $(5,8)$. In 
Table 1 Participant demographics

\begin{tabular}{|c|c|c|}
\hline Category & Characteristic & $\mathrm{N}[\%]$ \\
\hline \multirow[t]{3}{*}{ Current position } & Academic physician & 21 [75] \\
\hline & $\begin{array}{l}\text { Community/private } \\
\text { practice physician }\end{array}$ & $3[11]$ \\
\hline & Resident/fellow & $4[14]$ \\
\hline \multirow[t]{2}{*}{ Country of practice } & USA & 25 [89] \\
\hline & Canada & $3[11]$ \\
\hline \multirow{9}{*}{$\begin{array}{l}\text { Primary } \\
\text { subspecialty(s) }\end{array}$} & Palliative care & 14 [50] \\
\hline & Thoracic & $10[40]$ \\
\hline & General & 9 [32] \\
\hline & Gastrointestinal & 7 [25] \\
\hline & Genitourinary & 6 [21] \\
\hline & Central nervous system & $5[18]$ \\
\hline & Breast & $4[14]$ \\
\hline & Head and neck & $3[11]$ \\
\hline & Other & 8 [29] \\
\hline \multirow{4}{*}{$\begin{array}{l}\text { Dedicated palliative } \\
\text { care physicians at } \\
\text { institution* }\end{array}$} & 0 & $2[7]$ \\
\hline & $1-2$ & 8 [29] \\
\hline & $3-4$ & 7 [25] \\
\hline & 4 or more & 11 [39] \\
\hline \multirow{3}{*}{$\begin{array}{l}\text { Dedicated palliative } \\
\text { radiation oncologists } \\
\text { at institution* }\end{array}$} & 0 & 17 [61] \\
\hline & $1-2$ & $6[22]$ \\
\hline & 3 or more & $5[18]$ \\
\hline
\end{tabular}

*, the term 'dedicated' was used to describe a physician that specializes in palliative care or palliative radiation oncology, respectively.

this study, we surveyed the Society of Palliative Radiation Oncology (SPRO) membership to identify barriers to appropriate referral for palliative RT that they encounter in their practice, and identify specific groups of physicians who the radiation oncologists believed would benefit most from further education on when to refer patients for this important aspect of supportive care.

\section{Methods}

After obtaining ethics approval from the West Virginia University institutional review board (expedited protocol number 1901437100), an electronic survey was sent to all 136 members of SPRO. This convenience sample was selected for participation because the investigators felt that they were likely to be more knowledgeable about palliative RT referral patterns than the average radiation oncologist who has less of an interest in this particular patient population. Research Electronic Data Capture (REDCap), a secure web application used to build and manage online surveys and databases, was used to develop and disseminate the surveys. Two emails were sent to the SPRO membership soliciting participation. All responses were received between November 2018 and January 2019. Participation was anonymous, completely voluntary, and no financial incentive was provided.

The survey consisted of five sections. Section one assessed whether patients with newly diagnosed spinal cord compression, brain metastasis, thoracic airway obstruction, esophageal obstruction, and urinary obstruction were more commonly referred first to radiation oncology, the appropriate surgeon/procedure-oriented subspecialist, or both simultaneously at participants' institutions. Section two assessed in a multiple choice format the perceived most important and second most important barriers to referral for medical oncologists, surgeons, palliative care physicians, and primary care physicians. Section three assessed the perceived frequency of delays in referrals and potential reasons for those delays. Section four assessed the perceived level of understanding of different types of referring physicians of the risks and benefits of palliative RT on a 9-point Likert-type scale ( $1=$ not at all knowledgeable, 9= as knowledgeable as a radiation oncologist). Section five collected demographic information about the participants and their institutions. All survey questions and answer choices are shown in Table S1. Descriptive statistics including mean, standard deviation (STD), median, and interquartile range (IQR) were used to summarize the findings.

\section{Results}

A total of 28 radiation oncologists responded to the survey (response rate 20.5\%). The demographics of these participants are shown in Table 1. Eighty-nine percent of participants had at least one palliative care physician readily available to them at their primary practice location. The majority of participants also had readily available gastroenterology (89\%), interventional radiology (86\%), pain medicine $(82 \%)$, spine surgery $(82 \%)$, thoracic surgery/interventional pulmonology (82\%), urology (79\%), 


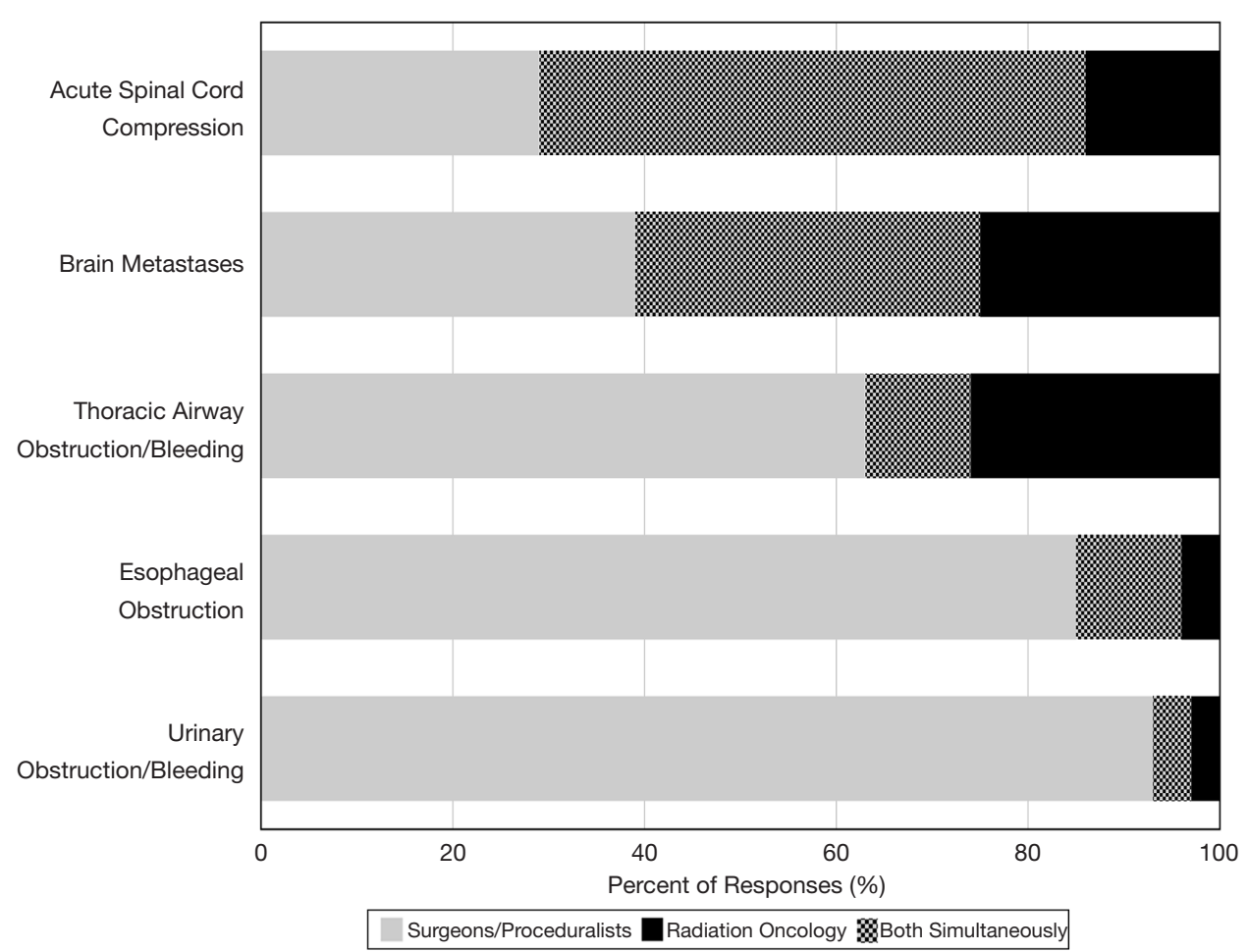

Figure 1 Participants were asked which type of physician most commonly receives the initial referral for patients diagnosed with several common indications for palliative radiation therapy.

and neurosurgery (79\%).

Figure 1 shows the perceived order of referrals for five common clinical indications for palliative RT. It was felt that radiation oncology was consulted first or simultaneously with spine surgery or neurosurgery more than $50 \%$ of the time for both spinal cord compression and brain metastases, respectively. However, it was felt that radiation oncology was much less likely to be part of the initial consultation for thoracic airway obstruction/bleeding, esophageal obstruction, or urinary obstruction/bleeding, which were more often initially evaluated by thoracic surgery/interventional pulmonology, gastroenterology, and urology, respectively.

Figure 2 shows participant views of how knowledgeable various medical and surgical subspecialists were about palliative RT. Neurosurgeons, head and neck, thoracic, and gynecologic surgeons were felt to be most knowledgeable among the surgical subspecialists, whereas palliative care and medical oncology were felt to be most knowledgeable among the medical subspecialists. Of note, primary care, geriatric medicine, and emergency medicine were considered to be among the least knowledgeable.
Figure 3 summarizes participants' perceptions of the two most common barriers to referrals for surgeons, palliative care physicians, and primary care physicians. The most common barriers to referral for primary care physicians and surgeons was thought to be a lack of knowledge about the benefit of palliative RT, and that it was the responsibility of the medical oncologist to do it. The palliative care physicians were thought to be more concerned with patient convenience, performance status, and other factors like cost, delay in hospice enrollment, and reimbursement concerns if a patient is on hospice. For medical oncologists (not pictured in the figure), participants felt that the greatest barriers were potential interference with systemic therapy (33\%), lack of knowledge about the benefit of palliative RT (28\%), and toxicity concerns (22\%).

On average, participants felt that referrals for palliative RT were inappropriately delayed $46.5 \%$ (STD 20.2\%) of the time. Similarly, waiting for tissue confirmation of malignancy was perceived to delay radiation oncology consultation for symptom palliation on average $51.5 \%$ (STD $27.8 \%$ ) of the time when no prior biopsy had been obtained. Additional reasons for delayed referrals cited by participants 


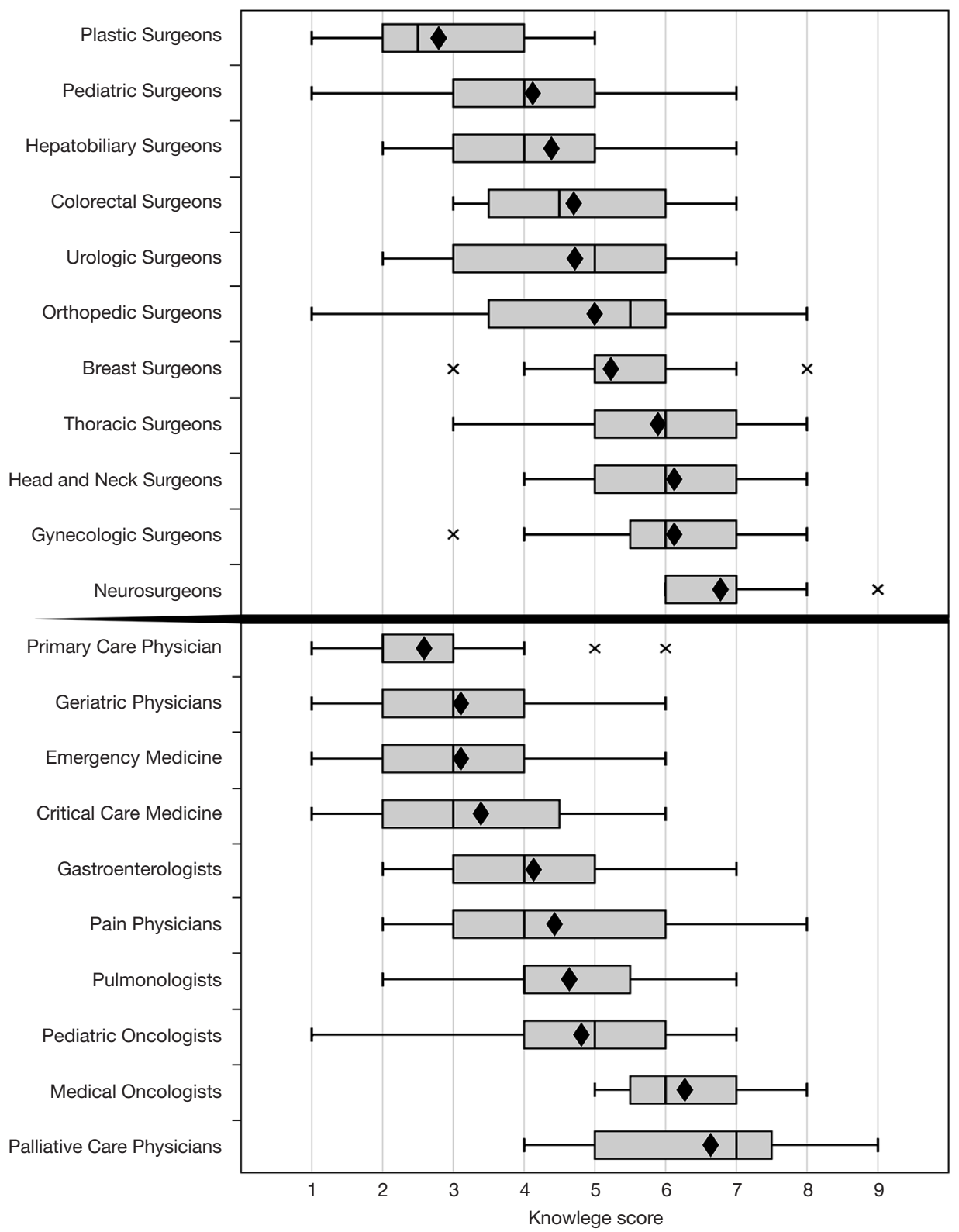

Figure 2 Box and whisker plot depicting the participants' perceived knowledge on a Likert-type scale from 1 (not at all knowledgeable) to 9 (as knowledgeable as a radiation oncologist) of various surgical and medical subspecialties. The boxes represent the interquartile range, vertical lines represent the median rating, and diamonds represent the mean rating. Extreme outliers were defined as greater than 1.5 times the value of the closest quartile.

included a cumbersome triage system between the different oncologists, other interventional services getting the referral first, referring physicians lacking an understanding of how imaging findings correlate with symptoms, concern that RT will delay systemic therapy by taking too long to start or finish, and an over-optimism that systemic therapy will work quickly enough or sufficiently enough to obviate the need for RT.

\section{Discussion}

In this study we have collected information from a group 


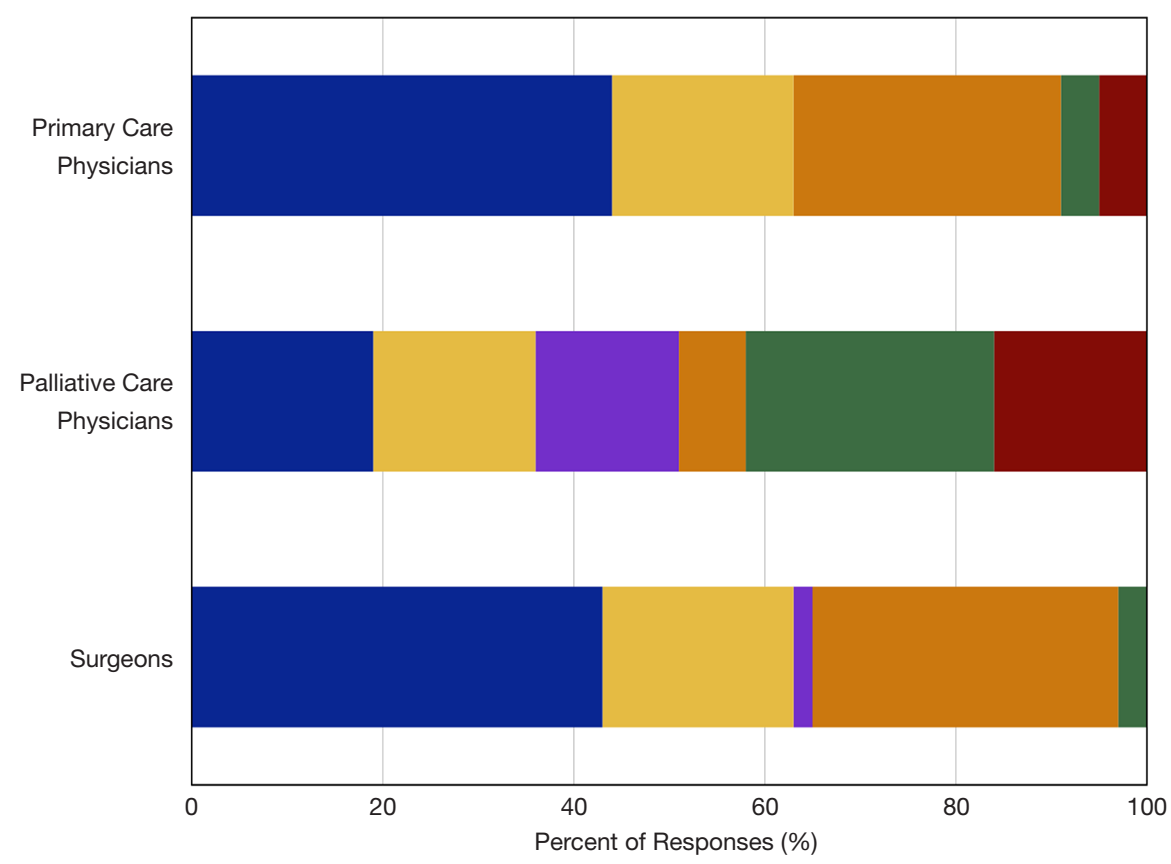

Figure 3 Most common perceived barriers to physician referral for palliative radiation therapy, including lack of knowledge on benefits of palliative RT (blue), concern for toxicity (yellow), concern for patient performance status (purple), responsibility of medical oncologist to refer (orange), concern for patient convenience (green), and other (red). RT, radiation therapy.

of radiation oncologists with an interest in palliative care, to identify perceived barriers hindering their ability to deliver timely and effective palliative RT at their institution. We found that nearly half of referrals for palliative RT were thought to be inappropriately delayed, radiation oncology is often a secondary consultation for certain conditions that are common indications for palliative RT, and the most important barriers to referral may vary considerably for different subspecialists. Overall, our data presents a number of hypothesis-generating observations that may help guide approaches to improve referral patterns for palliative RT.

Lack of knowledge about the benefits of palliative radiation was cited by $51 \%$ of participants as the most important barrier to referral across all categories of physicians that were asked about in this study. As such, education should be at the forefront of interventions to improve referral patterns. Targeting educational efforts towards physicians at the initial triage point in the outpatient and inpatient settings may be most effective, particularly as primary care, geriatric medicine, and emergency medicine, who are highly likely to see patients with cancer in some capacity, were considered to be among the least knowledgeable about palliative RT in our survey. An important message to these groups, as well as medical oncologists, may be that a referral is only a request for an opinion rather than an order for treatment, that early referral is encouraged, and ultimately the decision if, and when, to offer RT would involve shared decision-making between all of the oncologists involved. Hopefully this approach would also decrease reliance on medical oncologists to make all cancer-oriented referrals. Educational initiatives among primary care providers are likely to increase referrals for palliative RT in the USA, as has been demonstrated in two prior Canadian studies $(4,9)$. Finally, another important aspect of education is to accurately and realistically describe short- and longterm toxicities of radiation, so that other physicians can understand that although curative-intent radiation can cause late toxicities like bleeding, obstruction, or pain, radiation is also very good at alleviating these same symptoms in the palliative setting.

Educational initiatives would probably translate not only into more referrals, but perhaps more importantly earlier referrals. Ideally, patients would be treated before symptoms are so severe that they cannot tolerate the procedural aspects of delivering radiation (e.g., lying flat or wearing a head mask), and when patients still have 
sufficient lifespan to fully benefit from the radiation. Murphy et al. showed that in the USA approximately onefifth of cancer patients died within 2 weeks of receiving palliative RT, and one-third within one month of it, whereas Gripp et al. showed that among patients who died within 2 weeks of receiving palliative RT, only $26 \%$ had stable or improved symptoms from the radiation $(5,7)$. Physicians are notorious for overestimating survival towards the end of life, but regardless, the earlier that radiation is delivered, the increased likelihood patients are alive long enough to benefit from it (10). Earlier referral has the additional benefit of potentially sparing patients invasive procedures that may be less effective or have more potential for complications than RT (11-13).

Another initiative that is likely to improve referral patterns for palliative RT is for radiation oncologists to be more enthusiastic about using shorter courses of radiation when possible in order to minimize time off systemic therapy and any inconvenience for patients coming for daily treatment. Guadagnolo et al. demonstrated that in the USA fewer than $10 \%$ of patients received single fraction palliative RT for any indication near the end of life, despite its comparable efficacy in many cases (14-16). Considering institutional improvements for how patients are triaged, and engaging in multidisciplinary clinics whenever possible, is also likely to streamline the referral process.

The primary limitation of this study is selection bias, which may have affected our findings due to the relatively low response rate and convenience sample of potential participants who had a baseline interest in palliative care. On the other hand, because this population of physicians is actively engaged in providing palliative RT in their practice, our data is probably more accurate than if we were to have surveyed the general radiation oncology community at large. It is likely that our participants have somewhat more robust palliative radiation oncology programs than the average community radiation oncologist, and our findings may actually underestimate the extent of the problems with palliative RT referral identified. Another potential limitation is that we did not survey the other specialists themselves about their perspective on palliative radiotherapy, which may have provided some difference in outcomes compared to the radiation oncologists' perceptions of their colleagues. Despite these flaws, we believe that our findings do support greater attention to initiatives to improve palliative RT referral patterns in the future. Ideally such initiatives would be investigated in clinical trials to assess the clinical benefit of earlier radiation oncology referral for palliative RT.

\section{Acknowledgments}

None.

\section{Footnote}

Conflicts of Interest: The authors have no conflicts of interest to declare.

Ethical Statement: The authors are accountable for all aspects of the work in ensuring that questions related to the accuracy or integrity of any part of the work are appropriately investigated and resolved. After obtaining ethics approval from the West Virginia University institutional review board (expedited protocol number 1901437100).

\section{References}

1. Lutz S, Korytko T, Nguyen J, et al. Palliative radiotherapy: when is it worth it and when is it not? Cancer J 2010;16:473-82.

2. Jones JA, Lutz ST, Chow E, et al. Palliative radiotherapy at the end of life: a critical review. CA Cancer J Clin 2014;64:296-310.

3. Ashby M. The role of radiotherapy in palliative care. J Pain Symptom Manage 1991;6:380-8.

4. Fairchild A, Ghosh S, Baker J. Patterns of referral and knowledge of palliative radiotherapy in Alberta. Can Fam Physician 2012;58:e113-22.

5. Murphy JD, Nelson LM, Chang DT, et al. Patterns of care in palliative radiotherapy: a population-based study. $\mathrm{J}$ Oncol Pract 2013;9:e220-7.

6. Wong J, Xu B, Yeung HN, et al. Age disparity in palliative radiation therapy among patients with advanced cancer. Int J Radiat Oncol Biol Phys 2014;90:224-30.

7. Gripp S, Mjartan S, Boelke E, et al. Palliative radiotherapy tailored to life expectancy in end-stage cancer patients: reality or myth? Cancer 2010;116:3251-6.

8. Lloyd S, Dosoretz AP, Yu JB, et al. Academic and Resident Radiation Oncologists' Attitudes and Intentions Regarding Radiation Therapy near the End of Life. Am J Clin Oncol 2016;39:85-9.

9. Olson RA, Lengoc S, Tyldesley S, et al. Relationships between family physicians' referral for palliative radiotherapy, knowledge of indications for radiotherapy, 
and prior training: a survey of rural and urban family physicians. Radiat Oncol 2012;7:73.

10. Glare P, Virik K, Jones M, et al. A systematic review of physicians' survival predictions in terminally ill cancer patients. BMJ 2003;327:195-8.

11. Francis SR, Orton A, Thorpe C, et al. Toxicity and Outcomes in Patients With and Without Esophageal Stents in Locally Advanced Esophageal Cancer. Int J Radiat Oncol Biol Phys 2017;99:884-94.

12. Moeller B, Balagamwala EH, Chen A, et al. Palliative thoracic radiation therapy for non-small cell lung cancer: 2018 Update of an American Society for Radiation Oncology (ASTRO) Evidence-Based Guideline. Pract Radiat Oncol 2018;8:245-50.

13. Tsao MN, Rades D, Wirth A, et al. Radiotherapeutic

Cite this article as: Parker SM, Wei RL, Jones JA, Mattes MD. A targeted needs assessment to improve referral patterns for palliative radiation therapy. Ann Palliat Med 2019;8(4):516522. doi: 10.21037/apm.2019.08.02 and surgical management for newly diagnosed brain metastasis(es): An American Society for Radiation Oncology evidence-based guideline. Pract Radiat Oncol 2012;2:210-25.

14. Guadagnolo BA, Liao KP, Elting L, et al. Use of radiation therapy in the last 30 days of life among a large population-based cohort of elderly patients in the United States. J Clin Oncol 2013;31:80-7.

15. Lutz S, Berk L, Chang E, et al. Palliative radiotherapy for bone metastases: an ASTRO evidence-based guideline. Int J Radiat Oncol Biol Phys 2011;79:965-76.

16. Maranzano E, Trippa F, Casale $M$, et al. 8Gy singledose radiotherapy is effective in metastatic spinal cord compression: results of a phase III randomized multicentre Italian trial. Radiother Oncol 2009;93:174-9. 
Table S1 Survey questions and answer choices

Question
Section 1: pathway of referrals
A-E (multiple choice): to which service are patients with newly diagnosed (A) acute spinal cord compression,
(B) brain metastases, (C) thoracic airway obstruction, (D) esophageal obstruction, or (E) urinary obstruction
referred first?
Section 2: barriers to referrals
A-B (multiple choice): what is the most important (A) primary and (B) secondary barrier preventing medica
oncologists from referring more patients for palliative radiation therapy?
oncologists from referring more patients for palliative radiation therapy?

A-C (multiple choice): what is the most important barrier preventing (A) surgeons, (B) palliative care physicians, and $(C)$ primary care physicians from referring more patients for palliative radiation therapy?

A-C (multiple choice): What is the second most important barrier preventing (A) surgeons, (B) palliative care physicians, and $(C)$ primary care physicians from referring more patients for palliative radiation therapy?

(free response): if you answered "other" to any above questions, please explain

Section 3: delays in referrals

(rating scale): what percentage of the time do you feel that the timing of consultation for palliative radiation therapy is inappropriately delayed?

(rating scale): among patients that have not yet had a biopsy to confirm a likely new diagnosis of Stage IV malignancy, what percentage of the time does obtaining that biopsy delay radiation oncology consultation for symptom palliation?

(free response): what other factors inappropriately delay referral for palliative radiation therapy?

Section 4: knowledge of referring physicians

A-J (Likert scale): please rate how knowledgeable each of the following types of physicians are in their understanding of the risks and benefits of palliative radiation therapy $-(A)$ medical oncologists, $(B)$ pediatric oncologists, (B) palliative care physicians, (D) emergency medicine, (E) pulmonologists, (F) gastroenterologists, (G) primary care physicians, $(H)$ critical care medicine, (I) geriatric physicians, $(J)$ pain physicians

A-K (Likert scale): please rate how knowledgeable you believe each of the following types of surgical oncologists are in their understanding of the risks and benefits of palliative radiation therapy related to their disease site-(A) neurosurgeons, (B) head and neck, (C) thoracic surgeons, (D) breast, (E) hepatobiliary, (F) colorectal, $(\mathrm{G})$ gynecologic, $(\mathrm{H})$ urologic, $(\mathrm{I})$ orthopedic, $(\mathrm{J})$ pediatric, $(\mathrm{K})$ plastic surgeons

Section 5: demographic information

(multiple choice): what is your current position?

(multiple choice): what is your current country of practice?

(numerical input): how many radiation oncologists are in your department?

(checked boxes): what are the primary disease site(s) that you treat?

(V) (checked boxes): what types of specialty services are available at your primary practice location?

(VI) A-B (multiple choice): how many dedicated (A) palliative care physicians or (B) palliative radiation oncologists work with cancer patients at your institution?
Answer choices

Specialist/proceduralist

Radiation oncology

Both Simultaneously

Other

Lack of knowledge about the benefit Concern about toxicity Concern about patient performance status Interference with systemic therapy Concern about patient convenience Other

Lack of knowledge about the benefit Concern about toxicity Concern about patient performance status Medical oncologists job to refer Concern about patient convenience Other

Lack of knowledge about the benefit Concern about toxicity Concern about patient performance status Medical oncologists job to refer Concern about patient convenience Other

Free response

0-100\%

0-100\%

Free response

Scale 1-9: 1= not at all knowledgeable; $9=$ as knowledgeable as a radiation oncologist

Scale 1-9: $1=$ not at all knowledgeable; $9=$ as knowledgeable as a radiation oncologist

Resident/fellow Academic physician Community/private practice physician Other

USA

Canada

Europe

Other

Numerical input

Refer to Table 1 for answer choices

Palliative care physician(s)

Interventional radiology

Pain physicians

Neurosurgery

Thoracic surgery/interventional pulmonology Gastroenterology Urology

Zero

One

Two

Three

Four

Five

Greater than five 